\title{
Changes in prescribing for bipolar disorder between 2009 and 2016: national-level data linkage study in Scotland
}

Laura M. Lyall*, Nagore Penades* and Daniel J. Smith

\section{Background}

People with bipolar disorder typically require long-term pharmacological treatment to prevent episodes of depression o mania. However, evidence-based guidelines are often not followed by prescribers and, in some countries, prescribing of lithium is in decline. Polypharmacy is also common in bipolar disorder.

\begin{abstract}
Aims
To employ a data linkage approach to describe and evaluate prescribing patterns in bipolar disorder in Scotland between 2009 and 2016.
\end{abstract}

\section{Method}

By linking prescribing data to the electronic Scottish Morbidity Records, we identified a cohort of 23135 patients with bipolar disorder who were prescribed psychotropic medication between 2009 and 2016. We examined trends in proportions of patients prescribed each of six drug categories. Random effects logistic models examined change in prescribing over years of interest.

\section{Results}

The most common form of treatment was antidepressant monotherapy (24.96\%), with only $5.90 \%$ of patients receiving lithium monotherapy. Prescribing of antipsychotics and antiepileptics increased from 2009 to 2016 (antipsychotics: odds ratio $1.16,95 \% \mathrm{Cl} 1.15-1.18$; anti-epileptics: odds ratio 1.34, 95\% Cl 1.32-1.36), whereas prescribing of lithium decreased (odds ratio $0.83,95 \% \mathrm{Cl} 0.82-0.85)$. Prescribing of valproate decreased from 2009-2016 in women, but increased in men (women: odds ratio $0.93,95 \% \mathrm{Cl} 0.90-0.97$; men: odds ratio $1.11,95 \% \mathrm{Cl}$ 1.04-1.18).

\section{Conclusions}

Antidepressant monotherapy was the most common form of treatment for bipolar disorder in Scotland and prescribing of lithium has declined between 2009 and 2016. The findings are concerning and represent a gap between treatment guidelines and clinical practice.

\section{Declaration of interest}

None.

\section{Keywords}

Prescribing; bipolar disorder; lithium; valproate; antidepressants

\section{Copyright and usage}

(c) The Royal College of Psychiatrists 2019. This is an Open Access article, distributed under the terms of the Creative Commons Attribution-NonCommercial-NoDerivatives licence (http://creativecommons.org/licenses/by-nc-nd/4.0/), which permits non-commercial re-use, distribution, and reproduction in any medium, provided the original work is unaltered and is properly cited. The written permission of Cambridge University Press must be obtained for commercial re-use or in order to create a derivative work.

\section{Pharmacological treatment for bipolar disorder}

Bipolar disorder is a severe affective disorder characterised by episodes of depression and mania or hypomania. It affects $1-2 \%$ of the global population and is associated with a wide range of adverse mental and physical health outcomes. ${ }^{1,2}$ Many individuals with bipolar disorder require long-term medication to treat and prevent episodes and to maintain mood stability.

Current pharmacological treatment options for bipolar disorder are broad and include mood stabilisers (e.g. lithium, sodium valproate), antipsychotics, antidepressants, hypnotics, anxiolytics and anti-epileptics. ${ }^{3}$ Despite the availability of newer treatments, lithium is still considered the most effective treatment for reducing recurrence of episodes and since 2014 has been recommended as a first-line treatment by the National Institute of Health and Clinical Excellence (NICE). ${ }^{3}$ Prior to this, 2006 guidelines recommended lithium, valproate or olanzapine (an antipsychotic) as first-line treatments. ${ }^{4}$ There is also evidence that lithium has a specific anti-suicidal effect and it may be associated with fewer long-term negative physical health outcomes than other psychotropic medications. ${ }^{5,6}$ Moreover, the risk to the fetus of intrauterine exposure to lithium as well as the long-term risk of renal failure in people treated with lithium are both lower than previously reported. ${ }^{7,8}$

* These authors contributed equally to this article.
Despite NICE recommendations, however, lithium remains under-prescribed in clinical practice. Indeed, bipolar disorder has been identified as one of the areas of psychiatry with the widest gap between evidence-based treatment and clinical practice. ${ }^{9}$

\section{Changes in prescribing for bipolar disorder}

Routine healthcare data linkage studies in several countries have identified important changes in the use of lithium over time. ${ }^{10,11}$ Using national-level data from Denmark, Kessing and colleagues recently assessed prescription data for bipolar disorder between 2000 and 2011. ${ }^{10}$ Of the four drug categories studied, lithium went from being the most common drug prescribed for bipolar disorder to the least common, and was replaced by the use of atypical antipsychotics. ${ }^{10}$ Similar findings have emerged in Sweden, where lithium prescriptions for bipolar disorder decreased from 2007 to $2013 .{ }^{12}$ In partial contrast with these studies, however, an Italian study found that a drop in lithium prescriptions from 2002-2006 was followed by an increase from 2006-2010, perhaps reflecting changing attitudes among health practitioners. ${ }^{13}$

There has also been an increase in polypharmacy for bipolar disorder in many countries. ${ }^{14}$ Although combination treatments can sometimes be indicated, 'irrational polypharmacy', with coprescription of medications that are redundant, inappropriate or even harmful is well documented. Overall, polypharmacy is thought 
to occur in up to $85 \%$ of people with bipolar disorder and has been linked to increased risk of medical comorbidities. ${ }^{14}$

\section{Aims}

It is currently unclear whether prescribing changes observed in other countries have also occurred in the UK in recent years. The excellent routine data linkage infrastructure in Scotland facilitates assessment of changes in patterns of prescribing for bipolar disorder over time at a population level. Here, we used a health informatics approach to conduct a Scottish national-level assessment of prescribing patterns for bipolar disorder between 2009 and 2016.

\section{Methods}

\section{Data sources}

In Scotland, publicly funded healthcare is administered through 14 National Health Service (NHS) health boards. In each health board, records of out-patient clinic attendance, general/acute hospital admissions and psychiatric hospital admissions have been recorded in Scottish Morbidity Records (SMR) since 1981. These SMR records contain data obtained through routine clinical encounters, including dates and duration of attendance/admission. Diagnoses are coded using International Classification of Diseases (ICD)-9 (1981) and ICD-10 (1995). Date and primary and secondary causes of death (ICD-9/ICD-10) have been recorded in the National Records of Scotland (NRS) deaths register since 1981. Since 1999, information on the date, number, strength, formulation and quantity of prescriptions dispensed in the community in Scotland has been recorded on a Prescribing Information System (PIS). ${ }^{15}$ Patient date of birth and gender are included in both SMR and PIS records, and ethnicity, marital status and Scottish Index of Multiple Deprivation (SIMD) score are sometimes recorded by health professionals.

In Scotland the use of a unique patient identifier, the Community Health Index number, facilitates linkage of routine health databases by the Information Services Division (ISD) of NHS Scotland. The ISD provides access to linked data sets by approved researchers via the National Services Scotland National Safe Haven and via four regional Safe Havens located within Aberdeen, Dundee, Edinburgh and Glasgow. These Safe Havens provide secure access to patients' clinical data as well as a research platform for the collation, management, dissemination and analysis of anonymised electronic patient records.

Working with the ISD, we used SMRs dating back to 1981 for hospital out-patient attendance (SMR00), general/acute hospital admission (SMR01) and psychiatric hospital admissions (SMR04) to identify a cohort of individuals in Scotland with a diagnosis of bipolar disorder (ICD-10 codes F30, F31, F38.0; ICD-9 codes 296.0-296.1, 296.4-296.89). All individuals in this cohort were linked to the PIS, available from 2009-2016 at the time data were obtained, to obtain information on the date and type of prescriptions received. From the PIS, data on prescriptions for lithium, valproate (both classified as 'mood stabilisers'), antipsychotics, antiepileptics, hypnotics, anxiolytics and antidepressants were extracted, as these reflect the most common categories of medication prescribed in bipolar disorder. ${ }^{16}$ In the analyses below, hypnotics and anxiolytics were collapsed into a single category, as in the British National Formulary chapter 4.1 'Hypnotics and anxiolytics. ${ }^{17}$ We had a particular interest in prescribing patterns for lithium over time (the first-line treatment according to NICE guidelines) so it was examined separately. Valproate was also examined separately to ascertain any changes in its use in response to recent guidelines relating to risks associated with fetal exposure. ${ }^{18}$

\section{Participants}

A cohort of 45276 patients with a diagnosis of bipolar disorder as defined by ICD codes was identified from SMR00, SMR01 and SMR04 records. Of this cohort, 23261 individuals, according to the PIS, received at least one prescription of any of the drug categories of interest (hypnotics/anxiolytics, antipsychotics, lithium, valproate, antidepressants or anti-epileptics) between 2009 and 2016. Of these, $126(0.5 \%)$ had ICD codes which are typically used to code bipolar disorder type II, or 'other/unspecified' bipolar disorders (ICD-10 F31.8; ICD-9 296.8). For greater consistency of bipolar types included, we excluded these patients to focus analyses on patients with ICD codes consistent with bipolar disorder type I $(n=23135)$. For the current analyses, when examining each year of interest separately, individuals were excluded if their earliest SMR record of bipolar disorder was during or after the year of interest, or if their year of death, if applicable, was during or before the year of interest. For some analyses, we collapsed across all years of interest: in these cases, the sample consisted of patients whose earliest SMR record of bipolar disorder was before 2009, whose record of death was after 2009 or not applicable and who received relevant prescription(s) in any year from 2009-2016.

It is not possible to ascertain reliably from the SMR/PIS data whether those individuals in the bipolar disorder cohort (identified through the SMR) who did not have any PIS records of any prescriptions (of the selected categories) were still resident and/or receiving treatment in Scotland for each year of interest. Individuals without records of relevant prescriptions may have been resident in Scotland but not receiving psychotropic medication (in the categories of interest), or may no longer be registered with the Scottish NHS and are either receiving treatment elsewhere or have died elsewhere. As a result, our analyses were focused on individuals with PIS records for the major medication categories of interest. We were unable to estimate how many individuals with bipolar disorder in Scotland were unmedicated or receiving other psychotropic medications.

For the majority of analyses, we applied the inclusion criterion that an individual must have been consistently prescribed the same medication for a minimum period of 3 months in the year of interest (see Medication variables).

\section{Medication variables}

Prescriptions were coded into six categories of medications: hypnotics/anxiolytics (British National Formulary subsection 4.1 'Hypnotics And Anxiolytics'), antipsychotics (British National Formulary chapter 4.2 'Drugs used in psychoses and related disorders', excluding lithium and valproate), lithium (British National Formulary subsection 4.2.3 'Drugs used for mania or hypomania', specific drug codes lithium carbonate or lithium citrate), valproate (British National Formulary subsection 4.2.3 'Drugs used for mania or hypomania', specific drug code valproic acid; or British National Formulary chapter 4.8 'Antiepileptic drugs', specific drug codes [semi]sodium valproate or valproic acid), antidepressants (British National Formulary chapter 4.3 'Antidepressant drugs') or anti-epileptics (British National Formulary chapter 4.8 'Antiepileptic drugs', excluding [semi] sodium valproate and valproic acid). ${ }^{17}$

For each year of interest, individuals were coded as having been prescribed a given drug category (from the categories listed above) for an estimated minimum 3 months if (a) they received at least four prescriptions of the same drug category within a year, and (b) the average interval between prescriptions was between 21 and 84 days. This interval was selected based on the estimated typical duration of chronically prescribed prescriptions of around 56 days (between 28 and 84 days): ${ }^{19}$ an 84 day window is typically 
considered a reliable metric of drug exposure; a 21 day lower cut-off was selected to allow for early collection of prescriptions. Coding was not mutually exclusive: the same individual could meet the criteria for more than one drug category. In the analyses below, 'treated' refers to patients meeting these criteria.

We also calculated individuals' most typical form of treatment, in the form of their modal combination of drug categories across all years of interest. We defined combinations of drug categories as those prescribed together on the same date, thus using a conservative definition of drug category polypharmacy. For prescriptions meeting the above 3 month minimum criteria, each patient's modal combination of drug categories prescribed on the same date was defined as their modal form of treatment. This modal combination could consist of only one drug category (referred to as 'monotherapy', although this may comprise more than one specific drug from the same category) or multiple types ('polypharmacy' here referring to prescription of more than one drug category).

The modal form of treatment was also calculated separately for each year from 2009 to 2016. We applied the criterion that the modal combination of drug types must make up at least one third of the individual's total prescriptions for a given year (distinct prescriptions counted as distinct dates on which prescriptions were received). This was to account for patients who were undergoing a change in their treatment regimen, resulting in 'trials' of different combinations. As an example, for an individual who received five prescriptions of lithium and an antidepressant (each time prescribed on the same date and meeting 3 month minimum criteria) and four of lithium monotherapy within a year, the lithium/antidepressant polypharmacy would be coded as their modal drug combination for that year.

\section{Demographic variables}

The bipolar disorder cohort comprised patients from all 14 NHS Scotland health boards (see Supplementary Table 1 available at https://doi.org/10.1192/bjp.2019.16). Because of very small numbers of patients for three island-based health boards (Orkney, Shetland and Western Isles), these three were combined for all analyses/ tables, both for ease of interpretation and to minimise risk of identifying individual patients.

Data on marital status and ethnicity were missing for large numbers of individuals, as these fields are not consistently completed at the time of clinic/hospital attendance. Two separate coding systems for marital status were employed within and between data sets. To maximise patients with available data, we recoded patients with the first, more detailed system into the second, simpler system, i.e. 'single', 'married or separated', 'widowed' and 'other' (including divorce, civil partnership). Ethnicity was recorded as 'White'; 'Asian', 'Black', or 'mixed race/other'. Because of small numbers in some categories, we recoded ethnicity as 'White' and 'other'.

SIMD scores provide an index of deprivation based on areabased measures of income, employment, education, housing, health, crime and geographical access. ${ }^{20}$ SIMD 2012 quintiles were used here, where one corresponds to the most deprived areas, and five to the most affluent.

Reported health board, marital status, SIMD quintile and even ethnicity in some instances changed within and/or between years. The modal value for each patient and year was therefore included in analyses conducted by year and, for tables/analyses reporting values across all years, the modal values across 2009-2016 were employed.

Using SMR00, SMR01 and SMR04 records, we identified the date and year when a diagnosis of bipolar disorder first appeared in each patient's records: this may not necessarily represent the date of first diagnosis. Date of death, where applicable, was extracted from NRS deaths records. These dates were used to exclude from analyses patients whose first record of bipolar disorder was in or after a given year of interest and/or whose year of death (if applicable) was in or before a year of interest.

\section{Data availability and ethical approval}

All authors had access to the study data via the National Services Scotland National Safe Haven. Data are not publically available as they contain potentially sensitive information. Applications for data access can be made via the ISD (http://www.isdscotland.org/ Products-and-Services/eDRIS/Data-for-Research/). The study was facilitated by eData Research and Innovation Service (eDRIS) under National Services Scotland's favourable ethical opinion from the East of Scotland NHS Research Ethics Service.

\section{Results}

\section{Most common forms of treatment}

Demographic characteristics (where available) are presented for all treated patients with bipolar disorder in Supplementary Table 1, for each (non-mutually exclusive) drug category. Table 1 presents the ten most frequent combinations of drug types received by patients across the period of interest (2009-2016). Of the 23135 individuals in the bipolar disorder cohort, 20796 (89.89\%) met criteria for being treated for at least 1 year from 2009 to 2016 . The most common form of treatment collapsing across all years of interest was antidepressant monotherapy (24.96\%), followed by antipsychotic monotherapy (12.94\%). Lithium monotherapy was the fifth most common form of treatment, with only $5.90 \%$ of treated patients receiving this as their modal treatment across the period 2009-2016.

The top ten most common forms of treatment separately for each year are displayed in Supplementary Table 2. The pattern was very similar to that for all years combined, with around 30$40 \%$ of patients receiving either antidepressant monotherapy or antipsychotic monotherapy as their modal form of treatment. However, the proportion receiving antidepressant or lithium monotherapy declined gradually from 2009 to 2016 .

Supplementary Table 3 shows the proportion of patients receiving monotherapy (i.e. a single drug category), polypharmacy of two distinct drug categories and polypharmacy of three or more drug categories in each year of interest. This table highlights that a slight decline in the proportion of patients receiving one or two categories of medication from 2009-2016 was countered by an

\begin{tabular}{|c|c|c|c|}
\hline Rank & Drug combination & $N^{\mathrm{a}}$ & $\%^{a}$ \\
\hline 1 & Antidepressant & 5191 & 24.96 \\
\hline 2 & Antipsychotic & 2690 & 12.94 \\
\hline 3 & Hypnotic/anxiolytic & 1436 & 6.91 \\
\hline 4 & Antidepressant and antipsychotic & 1287 & 6.19 \\
\hline 5 & Lithium & 1226 & 5.90 \\
\hline 6 & Anti-epileptic & 797 & 3.83 \\
\hline 7 & Valproate & 755 & 3.63 \\
\hline 8 & Antipsychotic and valproate & 635 & 3.05 \\
\hline 9 & Antidepressant and hypnotic/anxiolytic & 605 & 2.91 \\
\hline 10 & $\begin{array}{l}\text { Antidepressant, antipsychotic and hypnotic/ } \\
\text { anxiolytic }\end{array}$ & 552 & 2.65 \\
\hline Other & All other combinations & 5622 & 27.03 \\
\hline Total & & 20796 & 100.00 \\
\hline
\end{tabular}




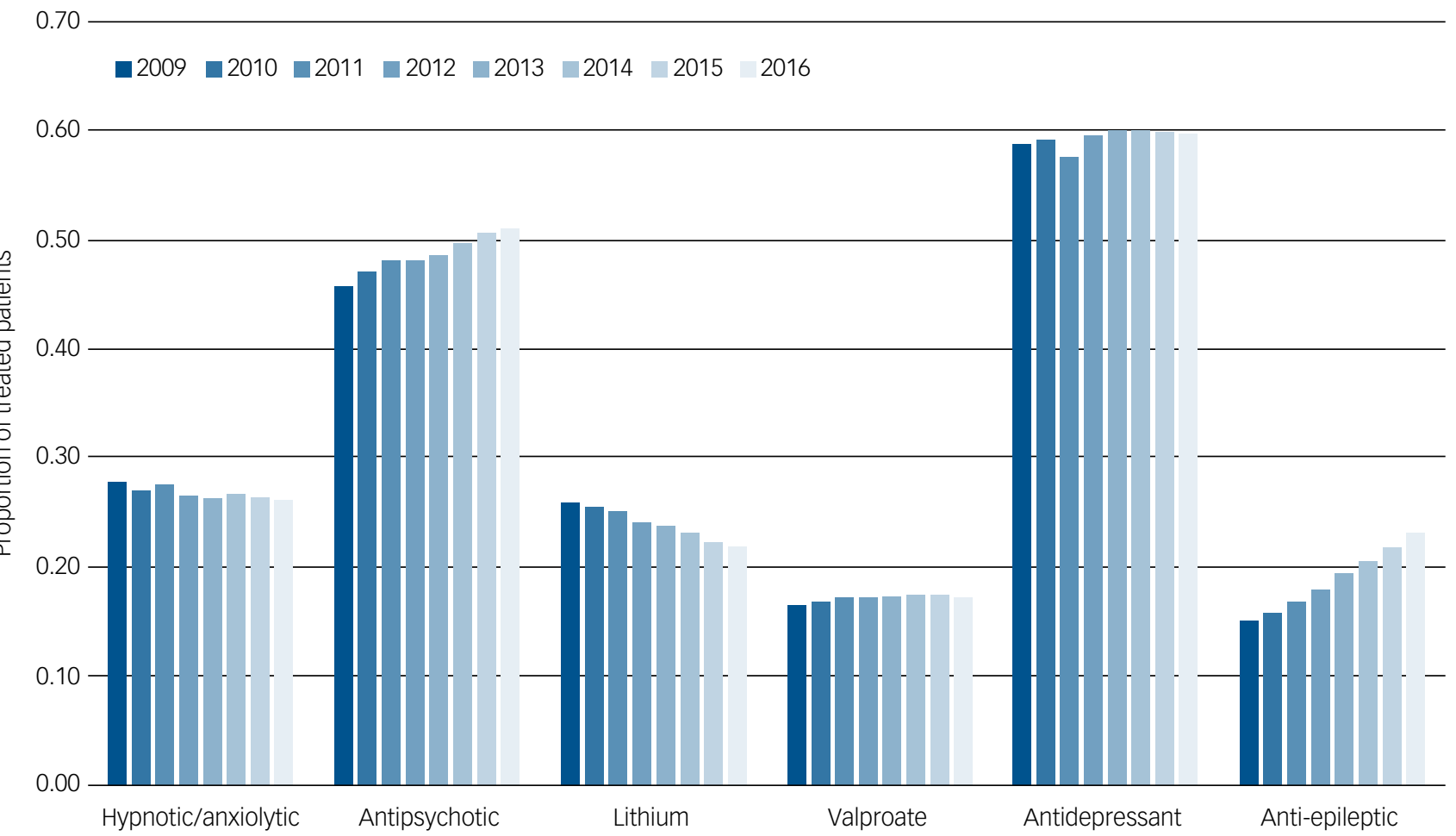

Fig. 1 Trends in the proportion of patients with bipolar disorder treated with each medication category across each year from 2009-2016. Data for each year include patients whose first Scottish Morbidity Record of bipolar disorder occurs before the year of interest and whose date of death (if applicable) occurs after the year of interest.

overall increase in patients prescribed three categories of medication. Trends in polypharmacy were then examined separately for combinations including and not including lithium. The proportion of patients prescribed combinations including lithium fell slightly from 14.08 to $11.94 \%$, whereas the proportion prescribed combinations not including lithium increased from 27.05 to $31.34 \%$.

\section{Trends in prescriptions of different drug categories by year}

The proportion of treated patients receiving each of the six categories of medication (whether alone or in combination with other drug categories) in each year from 2009 to 2016 is displayed in Fig. 1. There was a steady increase in the percentage of patients treated with antipsychotics (from 45.77 to $51.10 \%$ ) and anti-epileptics (from 15.20 to $23.24 \%$ ), alongside a decline in the percentage receiving lithium prescriptions (from 25.98 to $21.95 \%$ ). The overall percentage treated with antidepressants, hypnotics/anxiolytics and valproate remained relatively stable over time.

We were also interested in examining whether prescribing of valproate has declined in women of childbearing age over the 2009-2016 period, in light of guidance highlighting the risks associated with fetal exposure. Figure 2 shows an overall decline in prescriptions of valproate among women of childbearing age, whereas the proportion of men in the same age range prescribed valproate increased.

To examine change in the odds of prescriptions of each drug category across the years of interest, adjusted for available sociodemographic characteristics, we used random effects logistic models with standard errors clustered by patient. As data on sociodemographic variables such as ethnicity and marital status were missing for large numbers of patients, the models summarised in Table 2 adjusted only for age and sex to maximise numbers $(n=20300)$. Further models additionally adjusted for SIMD score, ethnicity, marital status and health board among the subset of patients with data on these covariates $(n=9322)$, and are presented in Supplementary Table 4. Multivariable random effects logistic models were employed as we were interested in examining the influence of time-invariant sociodemographic predictors (sex, ethnicity) on binary outcomes. As patients often showed little variability in predictors and outcomes across years of interest, it was not appropriate to use patients as their own controls as in fixed effects models. ${ }^{21}$

The odds of being prescribed antipsychotics, valproate and anti-epileptics all increased with each advancing year from 2009-2016 (Table 2); whereas the odds of treatment with lithium declined with increasing year (odds ratio 0.83 ; 95\% CI $0.82-0.85$ ). Increases in year were not reliably associated with prescriptions of antidepressants or of hypnotics/anxiolytics. In the fully adjusted models (Supplementary Table 4), odds ratios were slightly attenuated, but the significance and direction of results were unchanged.

Overall, odds of receiving valproate increased over the years of interest in both partially and fully adjusted models (partially adjusted: odds ratio 1.09; 95\% CI 1.07, 1.12; fully adjusted: odds ratio $1.09 ; 95 \%$ CI $1.06,1.12$ ). However, as we were interested in whether different trends were observed in women of childbearing age compared with men of a similar age, the model was repeated for valproate including a sex $\times$ year interaction term for individuals aged 18-50 years. Among the 8898 individuals included in this analysis (adjusted for age and sex), the interaction was significant (odds ratio 0.88 ; $95 \%$ CI $0.82-0.93 ; P<0.001$ ) and so stratified analyses examined trends in women and men in the 18-50 year age range separately, adjusted for age. For men $(n=3407)$, each advancing year from 2009 to 2016 was associated with increased odds of being treated with valproate (odds ratio 1.11; 95\% CI 1.04-1.18; $P=$ $0.001)$; whereas for women of the same age range $(n=5491)$, each 


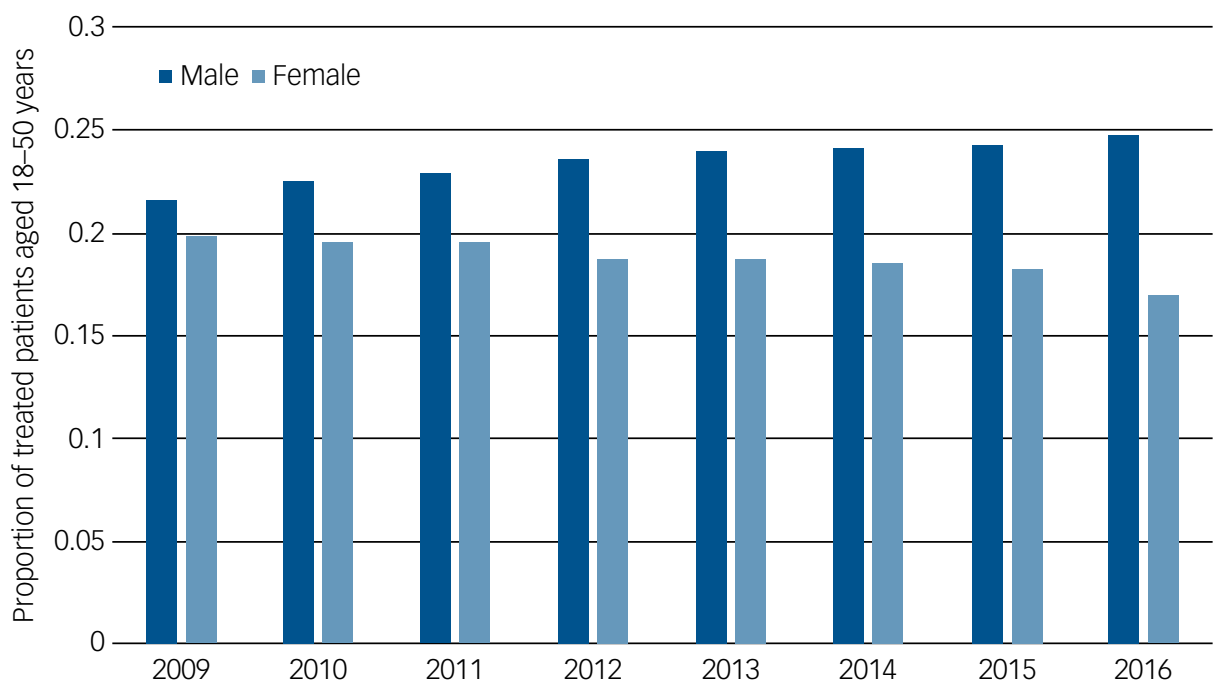

Fig. 2 Trends in the proportion of treated women with bipolar disorder of childbearing age (18-50 years) and men of the same age range treated with valproate across each year from 2009-2016. Data for each year includes patients whose first Scottish Morbidity Record of bipolar disorder occurs before the year of interest and whose date of death (if applicable) occurs after the year of interest.

year was associated with a decrease in odds of receiving valproate (odds ratio 0.93 ; 95\% CI 0.90-0.97; $P<0.001$ ).

\section{Discussion}

We sought to use large-scale, national-level routine health data in Scotland to investigate trends in prescribing for bipolar disorder between the years 2009-2016. Our findings demonstrate the feasibility of a routine data linkage approach and are of considerable clinical interest in light of treatment guidelines for bipolar disorder.

It is notable that antidepressant monotherapy was the most common form of treatment across all years, occurring in almost one in four patients with bipolar disorder. This is an area for concern because most treatment guidelines for bipolar disorder, including those from NICE in 2006 and 2014, caution against the use of antidepressants without mood stabiliser cover in bipolar disorder because of the risk of mood destabilisation and the potential to precipitate hypomania or mania., ${ }^{3,4}$ Although there is evidence to suggest antidepressants alongside mood stabilisers or antipsychotics are not associated with more bipolar disorder-related hospital readmissions, ${ }^{22,23}$ heightened risk of mania in patients with bipolar disorder treated with antidepressants as monotherapy has been clearly documented in large samples. ${ }^{23}$

The percentage of patients with bipolar disorder receiving lithium declined from $26 \%$ in 2009 to $22 \%$ in 2016 , and less than $6 \%$ of patients with bipolar disorder were on lithium monotherapy during this time. This low use of lithium and the trend towards decreasing lithium use is in contrast with the most recent NICE guidelines which encourage the first-line use of lithium and indeed state that all patients with bipolar disorder should be informed that lithium is the most effective long-term treatment. ${ }^{3}$ Clearly, the use of lithium in bipolar disorder can be complex (and not all patients will be suitable candidates), but overall these figures highlight a significant deviation between recommended treatment and clinical practice. Of note, recent data from England highlighted poor adherence to lithium monitoring guidelines by practitioners, ${ }^{24}$ further suggesting a need to improve competencies around lithium use. In contrast to the pattern for less year-on-year lithium prescribing, we found that the odds of being prescribed antipsychotics, valproate and anti-epileptics increased with each year between 2009 and 2016 .

It is likely that these trends in prescribing for bipolar disorder in Scotland are the result of a wide range of factors. Changes to medical training may have resulted in younger cohorts of psychiatrists being less likely to initiate lithium therapy. The pharmaceutical industry has also successfully promoted the use of alternatives. Other influences include the demise of traditional lithium clinics and the loss of Quality and Outcomes Framework incentives for lithium monitoring within primary care. ${ }^{25}$

We identified high rates of polypharmacy overall. In 2016, almost $26 \%$ of patients were treated concurrently with two classes of psychotropic medications and over $17 \%$ were treated with three or more (Supplementary Table 3). The proportion of patients prescribed three or more classes increased overall (from 15 to 17\%) between 2009 and 2016: the increase in polypharmacy appears to be most evident in drug combinations (of at least two drug categories) not including lithium, which increased gradually from 27 to $31 \%$ of the cohort, whereas polypharmacy including lithium fell (Supplementary Table 3). These trends suggest the increase in polypharmacy is linked to the declining use of lithium. Although many patients with bipolar disorder will require more than one class of medication (e.g. a mood stabiliser in combination with an antipsychotic), we suggest that the trend over time for a greater proportion of patients taking multiple classes of psychotropic medications is a major concern. This is particularly relevant given the high rate of cardiometabolic comorbidity observed in bipolar disorder. ${ }^{26}$

Despite previously being recommended as a first-line treatment option, valproate is not currently recommended for use in women of childbearing potential due to risks associated with fetal exposure. ${ }^{3,4}$ We found that the overall proportion of women prescribed valproate fell over time, likely reflecting better adherence to treatment guidelines and greater clinical awareness of potential risks. ${ }^{27}$

An important limitation in this study is that classification of individuals into the bipolar disorder cohort relied on the accuracy of the ICD code diagnoses recorded by healthcare professionals into electronic health records. Such records may be subject to administrative and diagnostic error, or early diagnoses may later be altered. However, a recent meta-analysis found the positive predictive value for bipolar disorder based on administrative data was moderately high at around $75 \%{ }^{28}$ 


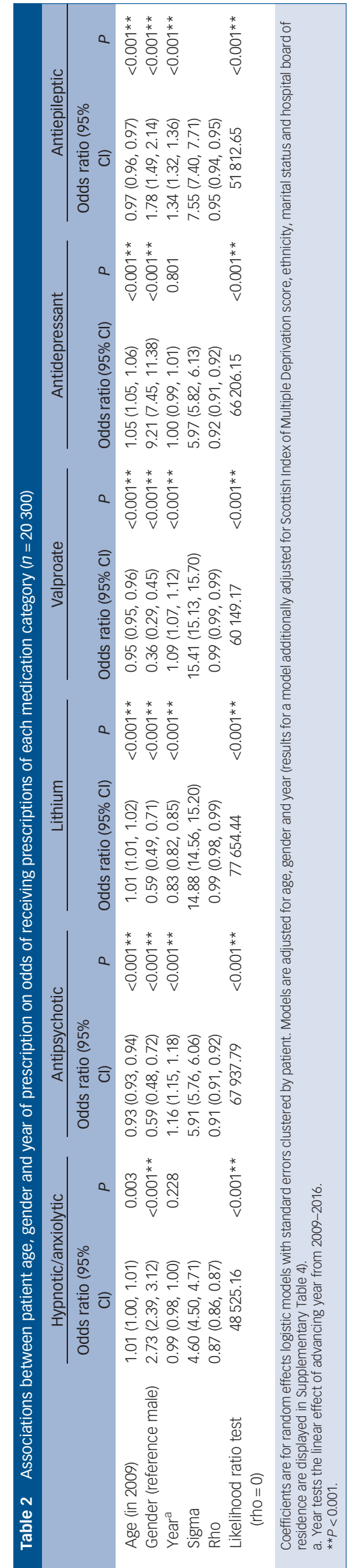

A further issue is that the nature of the routine data employed does not permit identification of which patients are no longer registered with the NHS in Scotland and who may be receiving treatment or have died elsewhere. We were unable to determine the proportion of patients with bipolar disorder in Scotland who are not treated in each year of interest. Inconsistent recording of sociodemographic data (e.g. marital status, ethnicity) also meant that these variables might not have been adequately controlled for.

We took the conservative step of excluding from analyses the small number of patients $(n=126)$ with ICD-9/-10 codes typically considered to reflect either bipolar disorder type II or other/unspecified bipolar disorder. ${ }^{29}$ The findings are therefore not relevant to prescribing patterns for bipolar disorder type II. In cohorts with larger numbers and/or more reliable distinction of bipolar disorder subtypes, it will be of interest to compare trends across subtypes and with disorders with overlapping symptoms such as schizoaffective disorder. Importantly, bipolar disorder type II is more often treated with antidepressants and less often with lithium compared with bipolar disorder type I. Exclusion of these participants here means that the observed trends towards decline in lithium and high use of antidepressants is unlikely to be driven by the bipolar disorder II subtype. Despite these limitations, this study demonstrates the feasibility of using Scotland's data linkage infrastructure to examine prescribing trends at a nationwide level.

Definitions of treated and polypharmacy vary widely between studies. ${ }^{14}$ A strength of this study is that relatively conservative criteria were used for each. Patients were defined as treated only in the presence of evidence of consistent use of a given drug category over at least 3 months; polypharmacy of drug categories was defined using evidence of consistent prescription of multiple drug categories on the same date. These definitions mean the observed trends likely reflect patients' established forms of treatment and are unlikely to be biased by brief trials of medications or by transitions between medications.

Although this study focused on data from NHS Scotland, these trends are likely to reflect practice across the UK and internationally. Bipolar disorder is a complex and highly morbid mood disorder but it can be managed effectively by combining medication with psychosocial approaches. Our findings suggest that most patients with bipolar disorder in Scotland are missing out on optimal treatments (such as lithium) and that many are receiving treatments (such as antidepressant monotherapy) that are at best ineffective and, at worse, detrimental for long-term outcome.

Laura M. Lyall, PhD (iD, Research Associate, Institute of Health and Wellbeing,

University of Glasgow, UK: Nagore Penades, MRCPsych, Consultant in General Adult University of Glasgow, UK; Nagore Penades, MRCPsych, Consultant in General Adult
Psychiatry, National Health Service Greater Glasgow and Clyde, UK; Daniel J. Smith, MD, FRCPsych (ID). Professor of Psychiatry, Institute of Health and Wellbeing, University of Glasgow, UK

Correspondence: Daniel J. Smith, Professor of Psychiatry, Institute of Health and Wellbeing, 1 Lilybank Gardens, University of Glasgow, Glasgow G12 8RZ, UK. Email: daniel.smith@glasgow.ac.uk

First received 5 Oct 2018, final revision 12 Nov 2018, accepted 17 Dec 2018

\section{Supplementary material}

Supplementary material is available online at https://doi.org/10.1192/bjp.2019.16.

\section{Funding}

This work was supported by a Medical Research Council Mental Health Data Pathfinder Award (reference MC_PC_17217) and a NHS Greater Glasgow and Clyde Partnership Award 2017. D.J.S. is supported by a Lister Prize Fellowship (2016-2021). 


\section{Acknowledgements}

We thank the eDRIS, part of NHS Scotland's ISD, for conducting data linkage and providing support with data access.

\section{References}

1 Goodwin FK, Jamison KR. Manic-Depressive Illness: Bipolar Disorders and Recurrent Depression (2nd edn). Oxford University Press, 2007.

2 Crump C, Sundquist K, Winkleby MA, Sundquist J. Comorbidities and mortality in bipolar disorder: a Swedish national cohort study. JAMA Psychiatry 2013; 70 : 931-9.

3 Kendall T, Morriss R, Mayo-Wilson E, Marcus E. Assessment and management of bipolar disorder: summary of updated NICE guidance. BMJ 2014; 349: g5673.

4 National Collaborating Centre for Mental Health. Bipolar Disorder: The Management of Bipolar Disorder in Adults, Children and Adolescents, in Primary and Secondary Care. British Psychological Society, 2006

5 Correll CU, Detraux J, De Lepeleire J, De Hert M. Effects of antipsychotics, antidepressants and mood stabilizers on risk for physical diseases in people with schizophrenia, depression and bipolar disorder. World Psychiatry 2015; 14: 119-36.

6 Cipriani A, Hawton K, Stockton S, Geddes JR. Lithium in the prevention of suicide in mood disorders: updated systematic review and meta-analysis. BMJ 2013; 346: $\mathrm{f} 3646$.

7 Cohen LS, Friedman JM, Jefferson JW, Johnson EM, Weiner ML. A reevaluation of risks of in utero exposure to lithium. J Am Med Assoc 1994; 271: 146-50.

8 Kessing LV, Gerds TA, Feldt-Rasmussen B, Andersen PK, Licht RW. Use of lithium and anticonvulsants and the rate of chronic kidney disease a nationwide population-based study. JAMA Psychiatry 2015; 72: 1182-91.

9 Fountoulakis KN, Vieta E, Sanchez-Moreno J, Kaprinis SG, Goikolea JM, Kaprinis GS. Treatment guidelines for bipolar disorder: a critical review. J Affect Disord 2005; 86: 1-10.

10 Kessing LV, Vradi E, Andersen PK. Nationwide and population-based prescription patterns in bipolar disorder. Bipolar Disord 2016; 18: 174-82.

11 Hayes J, Prah P, Nazareth I, King M, Walters K, Petersen I, et al. Prescribing trends in bipolar disorder: cohort study in the United Kingdom THIN primary care database 1995-2009. PLOS ONE 2011; 6: e28725.

12 Karanti A, Kardell M, Lundberg U, Landén M. Changes in mood stabilizer prescription patterns in bipolar disorder. J Affect Disord 2016; 195: 50-6.

13 Parabiaghi A, Barbato A, Risso P, Fortino I, Bortolotti A, Merlino L, et al. Lithium use from 2000 to 2010 in Italy: a population-based study. Pharmacopsychiatry 2015; 48: 89-94.

14 Fornaro M, De Berardis D, Koshy AS, Perna G, Valchera A, Vancampfort D, et al. Prevalence and clinical features associated with bipolar disorder polypharmacy: a systematic review. Neuropsychiatr Dis Treat 2016; 12: 719-35.

15 Alvarez-Madrazo S, McTaggart S, Nangle C, Nicholson E, Bennie M. Data resource profile: the Scottish National Prescribing Information System (PIS). Int J Epidemiol 2016; 45: 714-5f.

16 llyas S, Moncrieff J. Trends in prescriptions and costs of drugs for mental disorders in England, 1998-2010. Br J Psychiatry 2012; 200: 393-8.

17 British Medical Association, Royal Pharmaceutical Society of Great Britain. British National Formulary (March issue). BMJ Books and Pharmaceutical Press, 2014.

18 Nunes VD, Sawyer L, Neilson J, Sarri G, Cross JH. Diagnosis and management of the epilepsies in adults and children: summary of updated NICE guidance. BMJ 2012; 344: e281.

19 Guthrie B, Makubate B, Hernandez-Santiago V, Dreischulte T. The rising tide of polypharmacy and drug-drug interactions: population database analysis 19952010. BMC Med 2015; 13: 1-10.

20 Payne R, Abel G. UK indices of multiple deprivation - a way to make comparisons across constituent countries easier. Health Stat Q 2012; 53: 22-37.

21 Allison PD. Fixed Effects Regression Models. SAGE Publications, 2009.

22 O'Hagan M, Cornelius V, Young AH, Taylor D. Predictors of rehospitalization in a naturalistic cohort of patients with bipolar affective disorder. Int Clin Psychopharmacol 2017; 32: 115-20.

23 Viktorin A, Lichtenstein $\mathrm{P}$, Thase ME, Larsson $\mathrm{H}$, Lundholm C, Magnusson PKE, et al. The risk of switch to mania in patients with bipolar disorder during treatment with an antidepressant alone and in combination with a mood stabilizer. Am J Psychiatry 2014; 171: 1067-73.

24 Nikolova VL, Pattanaseri K, Hidalgo-Mazzei D, Taylor D, Young AH. Is lithium monitoring NICE? Lithium monitoring in a UK secondary care setting. J Psychopharmacol 2018; 32: 408-15.

25 Minchin M, Roland M, Richardson J, Rowark S, Guthrie B. Quality of care in the United Kingdom after removal of financial incentives. N Engl J Med 2018; 379: 948-57.

26 Martin DJ, Ul-Haq Z, Nicholl BI, Cullen B, Evans J, Gill JMR, et al. Cardiometabolic disease and features of depression and bipolar disorder: population-based, cross-sectional study. Br J Psychiatry 2016; 208: 343-51.

27 Virta $\sqcup$, Kälviäinen $R$, Villikka $K$, Keränen $T$. Declining trend in valproate use in Finland among females of childbearing age in 2012-2016 - a nationwide registry-based outpatient study. Eur J Neurol 2018; 25: 869-94.

28 Davis KAS, Sudlow CLM, Hotopf M. Can mental health diagnoses in administrative data be used for research? A systematic review of the accuracy of routinely collected diagnoses. BMC Psychiatry 2016; 16: 1-11.

29 James A, Hoang U, Seagroatt V, Clacey J, Goldacre M, Leibenluft E. A comparison of American and english hospital discharge rates for pediatric bipolar disorder, 2000 to 2010. J Am Acad Child Adolesc Psychiatry 2014; 53: 614-24. 